\title{
Management of ankylosing spondylitis case for ankle surgery under spinal anaesthesia by Taylor's approach.
}

\author{
Dr.Yoganarasimha.N ${ }^{1 *}$, Dr.Shivaramu.B. $T^{2}$, Dr.Shilpa Omkarappa ${ }^{3}$, \\ Dr.Radha.M.K ${ }^{4}$ \\ ${ }^{1}$ (Associate professor, Department of Anesthesiology, AIMS, Karnataka, India.) \\ ${ }^{2}$ (Assistant professor, Department of Anesthesiology, AIMS, Karnataka, India.) \\ ${ }^{3}$ (Postgraduate student, Department of Anesthesiology, AIMS, Karnataka, India.) \\ ${ }^{4}$ (Professor\&HOD, Department of Anesthesiology, AIMS, Karnataka, India.)
}

\begin{abstract}
Managing a case of ankylosing spondylitis can be difficult and challenging as both airway and vertebral column are deformed yet difficult and associated with extra articular manifestation. Taylor's approach can be considered ideally in these patients coming for lower limb surgeries. We present a young patient with severe ankylosing spondylitis with cardiac and pulmonary involvement coming for ankle surgery where we used Taylor's approach for successful central neuraxial blockade. By this approach the case was managed perioperatively optimally and safely.
\end{abstract}

Keywords: Ankylosing Spondylitis, Central neuraxial Bloackade, Taylors approach, paramedian technique, chronic inflammation

\section{Introduction}

Ankylosing spondylitis [AS] is a chronic inflammatory disease of axial skeleton and peripheral joints [1]. The inflammatory process usually begins at the sacroiliac joints and spread upwards to involve the spine and costo-vertebral joints leading to classical 'bamboo' spine.

The course of this disease is variable ranging from mild to severe disease involving extra articular manifestations like cardiovascular, ulcers, pulmonary fibrosis, uveitis.

Patient with deformed spine due to scoliosis, kyphoscoliosis, osteoarthritis, rheumatoid arthritis and ankylosing spondylitis are challenging to the anaesthesiologist due to technical difficulty for establishing the successful SAB because of reduced articular mobility, obliteration of the inter-spinal spaces and impossibility to position the patient adequately.

The paramedian technique may be selected in patient who cannot be positioned easily due to skeletal deformity and a modification of the paramedian technique by Taylor's approach has provided a reliable and less traumatic alternative in deformed spine for establishing the sub-arachanoid block. [2]

\section{Case Report}

A 14 year old female came to our hospital with complains of swelling over the right heel associated with difficulty in walking. On examination she has classical bamboo spine and exaggerated lumbar lordosis. Both elbow and shoulder extension was restricted. The disease was progressive and started 6 years back initially involves lower back gradually reaching the present stage. On cardiovascular examination she had mid systolic click, on further investigation -2D ECHO showed mitral valve prolapsed, her chest $\mathrm{x}$ ray showed multiple fibrosis shadow, she was diagnosed as severe ankylosing spondylitis with extra articular manifestations. Fig [1]

Airway was anticipated to be difficult because of the lack of extension of the cervical spine. A difficult airway cart was kept ready along with a flexible fiber-optic bronchoscope.

Written informed consent was obtained for both regional and general anaesthesia and the patient was kept NPO for 10 hours. The plan was to first attempt a spinal by Taylor's approach and in case of difficulty or failure alternate would be Epidural, GA or fibreoptic assisted intubation. Goal was to avoid bradycardia, LA toxicity and to maintain haemodynamic stability similar to pre operative values.

After arrival in the operation theater, standard monitoring including heart rate, ECG, pulse oxymetry and NIBP cycled at 3 min interval were done. A good peripheral line established with $18 \mathrm{G}$ i.v cannula, ringer lactate infusion started at a rate of $15 \mathrm{ml} / \mathrm{min}$.

In sitting position-Taylor's approach [modified paramedian technique at level of L5-S1] , a 23 G quince spinal needle inserted in a cephalomedial direction through a skin wheal raised $1 \mathrm{~cm}$ medial and $1 \mathrm{~cm}$ caudal to the posterior superior iliac spine into the subarachnoid space. A single attempt led to free flow of CSF and 1.8 $\mathrm{ml}$ of $0.5 \%$ heavy bupivacaine was injected table tilted laterally. Adequate level of block obtained up to T6 level over Right side and up to T8 level on the left, surgery started and the procedure lasted for 45 minutes. 
Patient did not require any airway intervention and her vitals were maintained as was the goal. Fig [2]

\section{Discussion}

The trend has been to deal with the airway challenge and avoid neuraxial anaesthesia in patients with AS, the reason being the inability to gain neuraxial access and the need for urgent airway control in case of complications of regional anesthesia.[3] However spinal anaesthesia is a acceptable alternative to general anaesthesia in the presence of ankylosing spondylitis for perineal or lower limb surgeries.[3,4] General anaesthesia in this patient has the risk of difficulty in managing airway, failed intubation and cervical spine injury. Fibreoptic bronchoscopy though available in our institution it was new to our department, also IPPV in this patient had the risk of rupturing the bullae in lungs and triggering cough reflex. Ankle block was avoided because there was swelling around the ankle and wanted to avoid local anaesthesia toxicity.

Subarachnoid block at L5-S1 interlaminar space offer distinct advantage. It is the lowest and widest space available so chances of trauma to spinal cord are negligible. This space is least affected by arthritic and degenerative changes, hence Taylor's approach is better alternative than the classical midline approach for establishing subarachnoid block with adequate sensory and motor blockade for the surgical procedure. Major advantage of the lateral approach is that no flexion of the back is required and there is minimal pain because though the ligaments are avoided and the sense of touch and needle control are more accurate, Kumar CM showed that spinal anaesthesia by the lateral approach is feasible and useful technique for lower limb surgeries in AS patients.[5]

Pre operative cardio respiratory evaluation is essential to determine the risk of valvular and conductive cardiac defects and also the risk of pulmonary disease. The sudden and intense reduction in systemic vascular resistance caused by spinal anaesthesia will not be tolerated by AS patients in case they had cardio pulmonary compromise.

Our patient had MVP + pulmonary fibrosis so low dose spinal drug was used and hemodynamic variability maintained near baseline levels. Possibility of requirement of ICD, emergency tracheostomy and post operative ventilator support was kept in mind and consent for the same was taken.

\section{IV.FIGURES}

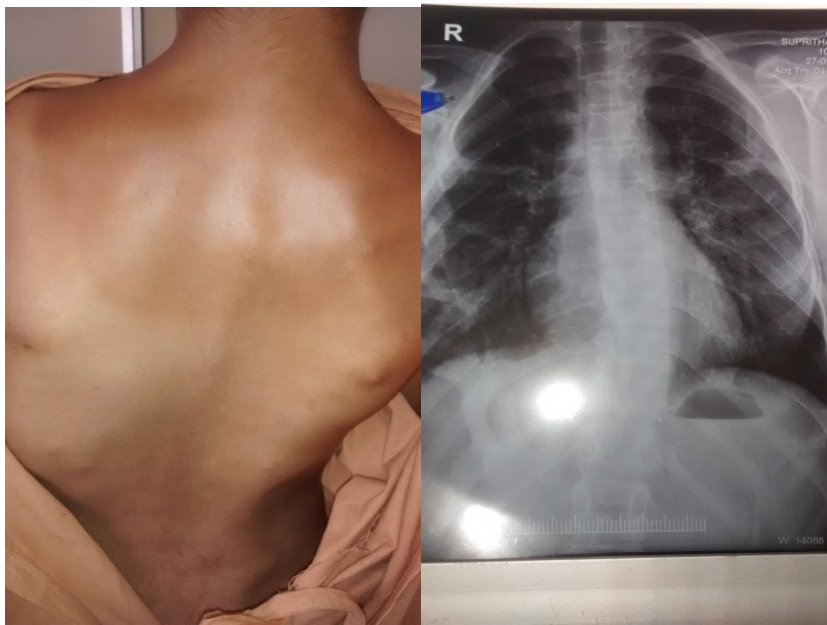

Fig [1]: x-ray showing narrowing of vertebral spaces "bamboo spine"
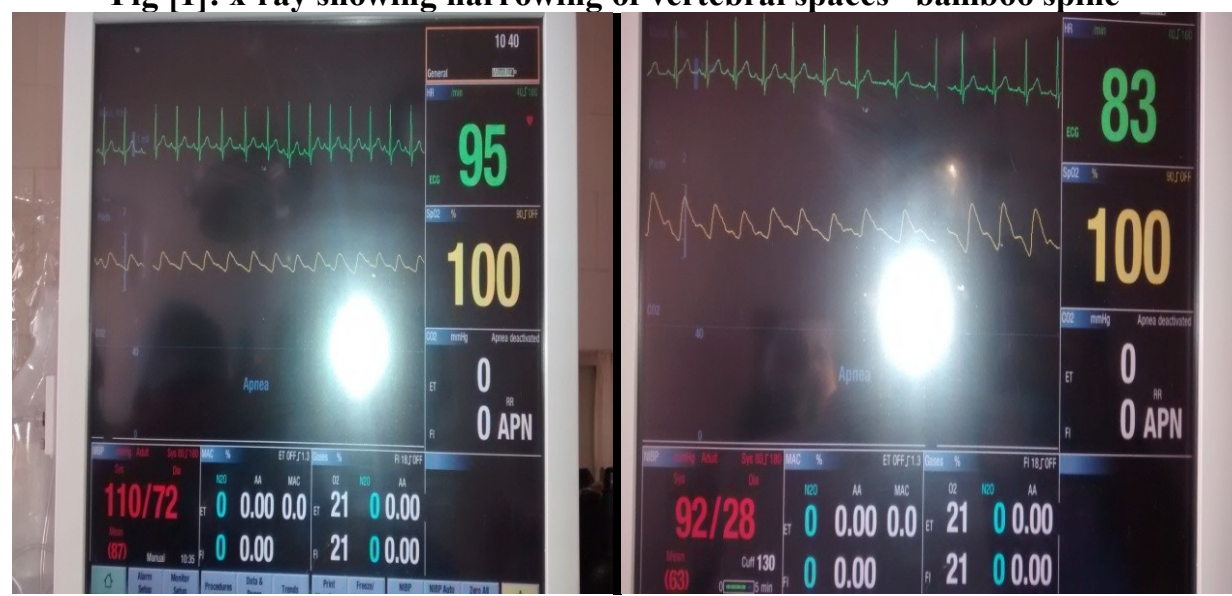

Fig [2]: Intra operative recording before and after SAB- showing stable vital parameters 


\section{Conclusion}

Subarachnoid anaesthesia has the potential to provide excellent operating conditions with fewer side effects, but patients with deformed spine represent challenges due to anatomical and technical difficulty for establishing successful Sub arachnoid block. Taylor's approach could provide a reliable and less traumatic alternative to the midline approach of lumbar puncture in deformed spine and as an alternative when general anaesthesia and conventional spinal anaesthesia seems to be technically impossible.

\section{References}

[1]. Goyal R, Singh S, Shukla RN, Singhal A. Management of a case of ankylosing spondylitis for total hip replacement surgery with the use of ultrasound -assisted central neuraxial blockade. Indian J Anaesth 2013;57:69-71

[2]. Gupta K, Rastogi B, Gupta PK, Rastogi A, Jain M, Singh VP. Subarachnoid block with Taylor's approach for surgery of lower half of the body and lower limbs: A clinical teaching study. Anaesth Essay Res 2012;6:38-41

[3]. NaliniKotekar et al. A case of severe ankylosing spondylitis posted for hip replacement surgery. Indian J.Anaesth 2007;51(6):546549 .

[4]. Woodward LJ, Kam PC. Ankylosing spondylitis: Recent developments and anaesthetic implications. Anaesthesia 2009;64:540-8.

[5]. Kumar CM, Metha M. Ankylosing spondylitis: lateral approach to spinal anaesthesia for lower limb surgery. Can J Anaesth 1995;42:73-6. 